\title{
VECTOR FIELDS GENERATING MORE THAN ONE FLOW
}

\section{SLAWOMIR DOROSIEWICZ* and KAZIMIERZ NAPIÓRKOWSKI*}

* Institute of Econometry, Central School of Planning and Statistics, Al. Niepodlegtosci 162, 02-519 Warsaw, Poland.

* Department of Mathematical Methods in Physics, Warsaw University, Hoza 74, 00-682 Warsaw, Foland.

(Received February 18, 1993 and in revised form July 19, 1993)

ABSTRACT. Vector fields generating more than one flow on a manifold are constructed. For one-dimensional case a complete description of the set of flows is given. For dimensions larger than one a method of constructing vector fields with dense or open branched sets sets 15 given. Density of vector fields with nonempty sets of branched points is studied.

KEY WORDS AND PHRASES. DYnamical systems, uniqueness, Cauchy problem.

1991 AMS SUBJECT CLASSIFICATION CODE: 34A10.

\section{INTRODUCTION.}

There are many elementary examples of vector fields for which the corresponding differential equation has non-unique solution of the Cauchy problem. The examples, as a rule, do not define flows on the underlying manifold. The reason is that considered vector fields have singularity at isolated points. In the present paper we show, mainly on examples, how to construct vector fields which generate more than one flow.

The basic sections in this paper are Section 3, 4 and 5. Section 3 describes the one-dimensional case. It occurs that the set of singularities ile. zeros) of the vector field has to be of special type, namely, it must contain the support of a nonatomic measure satisfying some additional conditions. The set of all flows generated by a given vector field can be completely described by means of class of measures supported on the set of zeros of the vector field.

Two and more dimensional case is considered in section 4. Situation is much more complicated. The points in which the non-uniqueness of the flow takes place (called branched points) are no longer zeros of the vector field. Another phenomenon is that the set of of branched points can be dense or open. We are not able to give a complete description as in Section 3, but some methods of constructing examples based on combining one dimensional examples with a constant vector field are given. Approximation methods developed in this section are use in Section 5 for a study of density properties of the set of vector fields generating more than one flow.

\section{GENERAL PROPERTIES OF FLOWS AND QUASI +LLOWS.}

DEFINITION 2.1

Let $X$ be a Hausdorff space.

(i) A continuous map $\varphi: \mathbb{R} \times X \rightarrow X$, such that $\varphi(O, x)=x$ for $x \in X$, satisfying the group property: $\varphi(t+s, x)=\varphi(t, \varphi(s, x))$ and for all $t, s \in \mathbb{R}$ and $x \in X$ will be called a flow on $x$. 
(11) If a map $\varphi: \mathbb{P} \times x \rightarrow x$ satisfies the group property and for all $x \in x$ the map $p(\cdot, x): \mathbb{R} \rightarrow x$ is continuous, then $p$ will be called a quas $-f$ low on $x$.

\section{DEFINITION $2 . \bar{Z}$}

Let $\varphi$ be a quasi-flow on a Mausdorff space $x$.

(1) The set $\{\varphi(t, x): t \in \mathbb{R}\}$ is called $\varphi$-orbz $t$ of $x$ and 15 denoted by $O_{\varphi}(x)$.

(11) A point $x \in X$ is called $\varphi-f 2 x e d$ point if $\varphi$-orbit of $x$ is trivial l.e. $0_{\varphi}(x)=\{x\}$. A set of $\varphi$-fixed points will be denoted by $S(\varphi)$.

EXAMPLE 2.3

For any given integer $n$, let $\varphi$ be the map $\mathbb{R}_{x} \mathbb{R}^{n} \rightarrow \mathbb{R}^{n}$ defined by $\varphi\left(t, x_{1}, \ldots, x_{n}\right)=\left(x_{1}+t, x_{2}, \ldots, x_{n}\right)$. It 15 easy to see that $\varphi$ is a flow on $\mathbb{R}^{n}$; we denote 1 t by 1 .

DEFINITION 2.4

Let $U$ be an open subset of a Hausdorff space $x$, and let $\phi, \psi$ be flows on $x$. We say that $\varphi$ and $\psi$ are equal on $U_{i f}$ the following condition is satisfied: for any $(t, x) \in \mathbb{R} x \cup$ if $\phi(t, x) \in U$ and $\psi(t, x) \in \cup$ then $\phi(t, x)=\psi(t, x)$.

DEFINITION 2.5

Let $X$ and $Y$ be Hausdorff spaces, UEX be a nonempty subset. Suppose that $\varphi$ and $\psi$ are the flows on $X, Y$ respectively. We say that $\varphi$ and $\psi$ are conjugate on $U$ if there exists a nomeomorphism $f: X \rightarrow Y$, such that:

$$
\psi(t, f(x))=f(\varphi(t, x))
$$

for all $x \in X$ and $t \in \mathbb{R}$ such that $\varphi(t, x) \in U$. If $U=M$, then we say that the flows $\varphi, \psi$ are conjugate.

Elementary properties of flows on one dimensional connected manifolds are described in the following two propositions.

PROPOSITION 2.6

Let $\varphi$ be a quasi-flow on $\mathbb{R}$. Then $\varphi$ is a flow on $\mathbb{R}$, and for any point $x \in \mathbb{R}$ exactly one of the following conditions is satisfied:

(a) $x$ is a $p$-fixed point,

(b) the orbit $O_{\varphi}(x)$ is an open interval containing the point $x$. Moreover, if a set 0 is a nontrivial $\varphi$-orbit then the restriction $\left.\varphi\right|_{\mathbb{R}_{\times} \mathbb{O}}$ of $\varphi$ to the set $\mathbb{R}_{\times} \mathcal{O}$ is conjugate with the unit flow $1_{1}$, i.e. there exists a homeomorphism $\sigma: \mathbb{R} \rightarrow 0$, which satisfies the equality: $\varphi(t, x)=\sigma\left(t+\sigma^{-1}(x)\right)$ for all $x \in \mathcal{O}$ and $t \in \mathbb{R}$.

A similar result can be formulated for the flows on the one dimensional sphere: PROPOSITION 2.7

Let $\varphi$ be a quasi-flow on $\mathcal{S}^{1}$, then $\varphi$ is a flow, and for any point $x \in \mathcal{S}^{1}$ exactly one of the following conditions is satisfied:

(a) $x$ is $\varphi$-fixed point,

(b) the orbit containing $x$ is equal $\$^{1}$,

(c) the orbit $O_{\varphi}(x)$ is diffeomorphic to $\mathbb{R}$, i.e. $O_{\varphi}(x)$ is an open arc.

The proof is elementary and will be omitted.

The following definition introduces a notion very useful in further considerations: the time measure of a flow on $\mathbb{R}$. This notion is a special case of the 
time measure ot a quasi-t low on a Hausdor ft" space (see [ż] $p .151$ ).

DEF INITION 2.8

Let $\beta$ denote the family of Borel sets on $\mathbb{R}$. and $\overline{\mathbb{P}}$ denote $\mathbb{R} U\{+\alpha\}$, let $\varphi$ be a fiow on $\mathbb{R}$. The $t$ ime measure of the flow $\varphi$ is a Borel measure $\mu: \beta \rightarrow \overline{\mathbb{P}}$ defined as follows:

(a) If $x$ is a $p$-fixed Doint then $\mu(\{x\})=+\infty$.

(b) $1+x \in 5(\varphi)$ then for any Borel subset $A=O_{\varphi}(x)$

$$
\left.\mu(A)=\mu_{L}(\varphi)^{-1}(A, x)\right)=\mu_{L}\left(\left\{\tau \in \mathbb{R}^{1}: \varphi(\tau, x) \in A\right\}\right)
$$

where $\mu_{L}$ is the Lebesque measure on the real line $\mathbb{R}$,

(c) for any Borel set A:

$$
\mu(A)=\sum_{(D \in \Gamma} \mu(\operatorname{ArO})+\mu(\operatorname{ArS}(\varphi)),
$$

where $\Gamma$ is the family of all nontrivial p-orbits.

PROPOSITION 2.9

$A$ necessary and sufficient condition for a Borel measure $\mu$ on $\mathbb{R}$ to be the time measure of a flow on $\mathbb{R}$ is that for each point $x \in \mathbb{R}$ exactly one of the following conditions is satisfied:

(1) $\mu(\{x\})=+\infty$,

(1i) there exist $\gamma, \delta$; $-\infty<\gamma<\delta \leq+\infty$, such that $x \in] \gamma, \delta[$ and the restriction of $\mu$ to the interval $] \gamma, \delta[$ is a nonnegative Borel measure with the properties:

1. If $A$ is a nonempty open subset of $] \gamma, \delta[$ then $\mu(A)>0$,

2. The measure of any compact subset of $] \gamma, \delta[$ is finite and $\mu(] \gamma, c[)=\mu(] c, \delta[)=+\infty$ for each $c \in] \gamma, \delta[$.

The proof is easy and will be omitted.

\section{VECTOR FIELDS AND FLOWS ON ONE-DIMENSIONAL MANIFOLDS.}

DEFINITION 3.1

Let $M$ be a $n$-dimensional smooth manifold, $V$ be a vector field and $\varphi$ be a flow on $M$. We say that $V$ generates the flow $\varphi$, if for any point $x \in M V(x)$ is the tangent vector to the curve $t \rightarrow \varphi_{t}(x)$ at $t=0$. The set of all flows generated by the vector field $V$ will be denoted by $F l(V)$.

An analogous definition may be formulated for quasi-flows. The set of all quasiflows generated by a vector field $V$ will be denoted by $F_{q}(V)$.

\section{DEFINITION 3.2}

Let $m$ be a cardinal number. We say that:

(i) a vector field $V$ on a differential manifold has Fm-property if the cardinality of the set $F l(V)$ is equal $\mathrm{m}$.

(1i) a flow $\varphi$ on differential manifold has Fm-property if it is generated by the vector field with Fm-property.

From standard theorems of the theory of ordinary differential equations follows that any smooth and bounded vector field on finite dimensional manifold generates exactly one flow, 1.e. it has F1-property. The following example shows that 
even on a one-dimensional manifold there exist vector tields which nave Fc-property.

EXMMIFLE క.:

Let $C$ denote the Cantor set in the interval [U. 1 J, and let $\partial: \mathbb{P} \rightarrow \mathbb{R}$. de a nonneuative continuous function on $F$ with period 1 such that $i x \in[0.1 j: \gamma(x)=(1)=[$. It is easy to see that the map

$$
\sigma: \mathbb{R} \rightarrow \mathbb{R}, \sigma(t)=\int_{0}^{t} \gamma(s) d s
$$

is a nomeomorphism of $\mathbb{R}$ onto $\mathbb{R}$. It is also obvious, that the velocity of the flow $\varphi=\sigma[1],, 1 . e . \varphi(t, x)=\sigma\left(t+\sigma^{-1}(x)\right)$ for $t \in \mathbb{R}$ and $x \in \mathbb{P}$, is equai

$$
v_{\varphi}(x)=\lim _{\varepsilon \rightarrow 0} \frac{\varphi(\varepsilon \cdot x)-x}{\varepsilon}=\frac{\partial p}{\partial t}(0, x)=\gamma\left(\sigma^{-1}(x)\right)
$$

for any point $x \in \mathbb{P}$. We shall show that the vector field $v_{\varphi}$ generates uncountable many flows on $\mathbb{R}$. Let $\mu_{\varphi}$ genote the time measure of the flow $\varphi$. The set $Z\left(V_{\varphi}\right.$ ' of critical points of $V_{\varphi}$ is equal:

$$
Z\left(v_{\varphi}\right)=\left\{x \in \mathbb{R}: \gamma\left(\sigma^{-1}(x)\right)=0\right\}=\left\{x \in \mathbb{R}: \sigma^{-1}(x) \in \underset{k \in \mathbb{Z}}{\bigcup} k+[C\}=\sigma\left(\bigcup_{k \in \mathbb{Z}} k+[)\right.\right.
$$

Let $v$ be a measure concentrated on the set $\sigma^{-1}, \bigcup_{k \in \mathbb{Z}} k+C$ ) of critical points of $v_{\varphi}$. One can take for example the measure generated by the Cantor function as follows. First, let us define a function $f: C \rightarrow[0,1]$ in the following way: if $r=\sum_{i=1}^{+\infty} r_{i} \cdot 3^{-i}$, where $r_{1} \in\{0,2\}$, then $f(r)=\sum_{i=1}^{+\infty} r_{i} \cdot 2^{-i}$. It can be shown that $f$ is a continuous sur jection, moreover, it has a unique extension to a continuous function $F$ on $[0,1]$. The derivative of this function vanishes on $[0,1]-C$, and does not exist on $C$. Let $F^{*}$ denote the following function: $F^{*}(x+k)=F(x)+k$ for all $x \in[0,1]$ and $k \in \mathbb{Z}$. It is obvious that the measure $v$ defined for any compact, nonempty interval $[\alpha, \beta]$ as:

$$
v([\alpha, \beta])=F^{*}(\sigma(\beta)) F^{*}(\sigma(\alpha))
$$

is a continuous nonnegative Borel measure concentrated on the set of critical points of the vector field $V_{\varphi}$. Let us return to general considerations of this example. It is easy to see that for any positive number $\tau$, the measure $\mu=\mu_{\varphi}+\tau \cdot \nu$ satisfies the conditions (1), (1i) of proposition 2.9 and therefore is the time measure of a flow a flow $\bar{\varphi}$ on $\mathbb{R}$. Now it is sufficient to show that this flow (i.e. the flow with the time measure $\mu$ is generated by the same vector field $v_{\varphi}$ i.e. the flows $\varphi$ and $\bar{\varphi}$ nave the same velocity $v_{\varphi}$.

1) The set $\mathbb{R}-Z\left(V_{\varphi}\right)$ is open. Consider any of its pounts $x$ and take a positive number $n>0$ such that $] x-2 n, x+2 R) d R-Z\left(V_{\varphi}\right)$. It means that $\mu \frac{-}{\varphi}([x, x+\varepsilon] \cup[x+\varepsilon, x])$ is equal $\mu_{\varphi}([x, x+\varepsilon] น[x+\varepsilon, x])$ for $\varepsilon<2 n$, and this fact 1 mplies the equality $\bar{\varphi}(t, x)=\varphi(t, x)$ in a neighbourhood of the point $t=0$. It means that the flows $\varphi$ and $\bar{\varphi}$ have the same velocities at the point $x$.

2) If $x \in Z\left(V_{\varphi}\right)$ then $V_{\varphi}(x)=0$, and $\lim _{\varepsilon \rightarrow 0} \frac{\varepsilon}{\mu_{\varphi}([x, x+\varepsilon] \cup[x+\varepsilon, x])}=0$

It follows from definition of the measure $\mu$ that 


$$
\lim _{\varepsilon \rightarrow 0} \frac{\left(\mu_{\varphi}+\tau \cdot \nu\right)([x, x+\varepsilon] \cup[x-\varepsilon, x])}{|\varepsilon|}=+\infty
$$

which is equivalent that the velocity of the flow $\bar{\varphi}$ at the point $\times$ vanishes. Therefore the vector field $v_{\varphi}=\gamma\left(\sigma^{-1}\right)$ generates the flows $\bar{\varphi}$ and $\varphi$.

In Section 4 we shall use vector fields with compact sets of critical points and similar properties.

EXAMPLE 3.4

Let $\gamma^{\prime}$ be any any smooth nonnegative function $\gamma^{\prime}: \mathbb{R} \rightarrow \mathbb{R}$, such that $\gamma^{\prime}=1$ on $\mathbb{R}-[-1 / 2,3 / 2]$ and $\gamma^{\prime}=\gamma$ on $[0,1]$, where $\gamma$ denotes the function from the last example. It is clear that the vector field $V^{\prime}=\gamma^{\prime}\left(\sigma^{\prime}, \mathbf{1}\right)$, where $\sigma^{\prime}(x)=\int_{0}^{x} \gamma^{\prime}(s) d s$, has the FC-property.

Example 3.3 suggests that there is a one to one correspondence between flows generated by a given vector field on $\mathbb{R}$ and some measures concentrated on the set of critical points of this field.

In further considerations, let $V$ be a continuous, bounded vector field on $\mathbb{R}$, and let $Z(V)$ denote the set of 1 ts critical points. First note that if $x_{0}$ is not a critical point of the vector field $V$ on $\mathbb{R}$, then there exists a neighbornood $U$ of $x_{0}$ such that the Cauchy problem

$$
\frac{d y}{d t}=v(y), \quad y(0)=x_{0}
$$

has exactly one solution in $U$.

Indeed, if $U$ is a connected component of $\mathbb{R}-Z(V)$ containing $x_{0}$, then we can write (3.1) on $U$ as: $t=t_{0}+\int_{x_{0}}^{y} \frac{d s}{v(s)}$, $y \in U$, obtaining exactly one function $t=t\left(\cdot, x_{0}\right)$ and equivalently one function $y=y\left(\cdot, t_{0}\right)$, which is the solution of $(3.1)$.

We shall consider now the set of flows generated by a given vector field $V$ on $\mathbb{R}$. Let $V$ be a continuous, bounded vector field on $\mathbb{R}$ and let $\varphi$ be a flow generated by this field with the time measure $\mu_{\varphi}$ and the set $S(\varphi)$ of $\varphi-f i x e d$ points. For any closed set $\Omega \subseteq Z(V)$ let $\left\{P_{\alpha}\right\}_{\alpha \in Q}$ be the family of connected components of the set $\mathbb{R}-\Omega$.

For further consideracions we introduce, for a given $\Omega$ and $\varphi$, the set $K_{\varphi}(\Omega)$, elements of which are all (not necessarily positive) Borel measures $m$ on $\mathbb{R}$ satisfying the following conditions:

1. $m$ is concentrated on $Z(V)$,

2. $\mu_{\varphi}+m$ is nonnegative and if $A$ is ary open nonempty subset of $\mathbb{R}$ then $\left(m+\mu_{\varphi}\right)(A)>0$,

3. If $A$ is a compact subset of any $P_{\alpha}$ then $m(A)<+\alpha$, and $\left(\mu_{\varphi}+m\right)\left(P_{\alpha}\right)=+\infty$,

4. for any $x \in Z(V) \lim _{\varepsilon \rightarrow 0} \frac{\varepsilon}{\left(m+\mu{ }_{\varphi}\right)(] x, x+\varepsilon[U] x+\varepsilon, x[)}=0$,

5. if $x \in \Omega$ then $m(\{x\})=+\infty$, for $y \in \Omega \mu(\{y\})=0$.

A topology in $K_{\varphi}(\Omega)$ may be introduced by the following 
DEFINITIUN 3.5

we say that a sequence $m_{n} \in k_{\varphi}(\Omega)$ converges to a measure $m$ if:

$$
\lim _{n \rightarrow+x} \sup _{x \in Q}\left\{m_{n}(B)-m(E) \mid: B \text { is any compact subset of } P_{x}\right\}=0
$$

The set $F I$ ( $V$ ) has the standard uniform convergence topology.

THEOREM 3.6

Let $V$ be a continuous, bounded vector field on $[P$. If the set $F 1(V)$ is not empty, then there exists a flow $\Phi \in f(V)$ such that the mapping

$$
\lambda: \bigcup_{\Omega} K_{\Phi}(\Omega) \rightarrow F I(V), \quad \lambda(m)=\Psi_{m}
$$

which assigns to a measure $m$ the flow $\psi_{m}$. which has the time measure $\mu_{\Phi}+m$, where $\mu_{\Phi}$ denotes the time measure of $\Phi$, is a continuous bi jection.

Proof. Since $F I(V) \rightleftharpoons$ we can choose $\varphi \in F l(V)$. For a closed set $\Omega \subset Z$ we denote by $F l(V, \Omega)$ the subset of $F l(V)$ consisting of all flows having $\Omega$ as the set of fixed points. It is clear that for $\Omega=S(\varphi)$ the mapping $\lambda$ gives a continuous bi jection between $k_{\varphi}(\Omega)$ and $F I(V, \Omega)$. To complete the proof it suffices to show that there exists a flow $\Phi \in f(V)$ with a minimal set of fixed points 1.e. $S(\Phi) S S(\psi)$ for any $\psi \in F l(V)$. We remark first that if $\varphi, \psi \in F l(V)$ and $\mu_{\varphi}, \mu_{\psi}$ are their time measures then $\mu=\min \left(\mu_{\varphi}, \mu_{\psi}\right)$ is the time measure of a flow belonging to $F l(V)$. We denote that flow by $\min (\varphi, \psi)$. Since $x \in S(\varphi)$ if and only if $\mu_{\varphi}(\{x\})=\alpha$ we have $\operatorname{Simin}(\varphi, \psi))=S(\varphi) r S(\psi)$. One can check that a decreasing net of time measures of flows in $F I(V)$ converges to the time measure of a flow in $F l(V)$. Using Kuratowski-Zorn lemma we get the existence of a flow $\Phi \in f l(V)$ with the minimal set of fixed points. Q.E.D.

An analogous result holds when $\mathbb{R}$ is replaced by $\mathbb{S}^{1}$. For manifolds of larger dimension than 1 one can conclude the following

COROLLARY 3.7

Suppose that $\varphi$ and $\psi$ are different flows on a manifold $M$, and each $\psi$-orbit is contained in a $\varphi$-orbit. If $\varphi$ and $\psi$ are generated by the same vector field $V$ then $\checkmark$ has at least one critical point.

\section{VECTOR FIELDS AND FLOWS ON HIGHER DIMENSIONAL MANIFOLDS.}

This part starts from the following definition describing singular points of a given vector field $V$ on finite-dimensional manifold.

DEFINITION 4.1

Let $V$ be a vector field on a differential manifold $M$.

(a) A point $x \in M$ will be called a $V$-strong branched point if there exist quasiflows $\varphi$ and $\psi$ generated by $V$ and a positive number $\varepsilon>0$ such that the set $R(\varphi, \psi)=\{t \in \mathbb{R}: \varphi(t, x) \psi(t, x)\}$ contains at least one of the intervals $(0, \varepsilon)$, $(-\varepsilon, 0)$. The set of $V$-strong branched points will be denoted by $R(V)$.

(b) A point $x \in M$ will be called a $V$-weak branched point if there exist two solutions $y_{1}(\cdot, 0, x), i=1,2$ of Cauchy Problem: 
such that $t=1)$ is a cluster point of the set $\left(t \in \mathbb{P}: v_{1}\left(t,(1, \cdots) x_{v_{2}}(t, 1) \cdot x\right)\right)$. Ine set of weak $V-t)$ ancher points $w_{1} 11$ de denoted by ${ }^{R}{ }_{w}(V)$.

It is obvious that each strong oranched point of a qiven vector fielo $v i 5$ I $v$-weak branched point, and $1 F \times$ is a $v$-weak oranrned point, then the Laucriv probiem (4.1) has uncountable manv soiutions (see for example [5], theorem 4.1) DEFINITION 4.:

If, for a given subset $A$ of a manifold M, there evists a vector field $V$ on in such that $K(V)=\hat{A}\left(R_{w}(V)=A\right)$, then $A w_{1} l i$ be called a strong branched set (resp. weak branched set).

The following fact follows easilv from Theorem 4.6:

FROFOSITION 4.3

Suppose that $V$ is a continuous bounded vector field on $R$. or $S^{1}$. then $R(V)$ is a nowhere-dense subset of this manifold, contained in the set of critical points of the field $\mathrm{V}$.

It follows from the above proposition that an open subset of $\mathbb{R}$ or $\$ \mathbf{S}$ can never be a weak branched set. Now we shall show that there exist strong branched sets dense in higher aimensional manifolds, especially in $\mathbb{P}^{2}$. The construction is a continuation of M. Lavrentiev's and P. Hartman's ideas (see [6] and [7]), who have constructed examples of ordinary differential equations with locally nonunique solution of Cauchy Problem in any point of an open subset of $\mathbb{R}^{2}$. It is equivalent to proving that $\mathbb{R}^{2}$ is a weak oranched set 1 .e. there exists a continuous vector field $V$ on $\mathbb{R}^{2}$ such that $R_{w}(V)=\mathbb{R}^{2}$. We are going to show more. namely, that $\mathbb{R}^{2}$ is a strong oranched set. First in the example 4.4 we construct a vector field $V$ such that $R(V)$ is dense in $\mathbb{R}^{2}$

EXAMPLE 4.4

We construct an example of a continuous vector field $V$ on $\mathbb{R}^{2}$, with a dense set of strong branched points. The construction is divided into two steps. In first of them we define an operation called MOD-operation and analyse its properties. The second step included a proper description of the construction. STEP 1

Let $v$ be a continuous vector field on $\mathbb{R}^{2}$ and $R(v)$ be the set of 1 ts strong branched points. Suppose that for a connected open subset $U$ of the set $\mathbb{R}^{2}-R(V)$ there exists a smooth local coordinate system $(\lambda, U)$ in which $v$ has the coordinates $\langle 1,0\rangle$.

Let $\Omega$ denote a nonempty rectangle $[a, b] \times[c, d] c a(U)$. We choose real functions $\alpha(\cdot)$ and $h(\cdot)$ such that $\alpha: \mathbb{R} \rightarrow \mathbb{R}$ is continuous in $] a, b[$, and $\alpha \equiv 0$ on $\mathbb{R}-] a, b[$, $h: \mathbb{R} \rightarrow] c, d\left[\right.$ is a diffeomorphism such that $\frac{d h}{d x} \rightarrow 0$ when $x \rightarrow \pm x$. We assume, moreover, that

$$
\int_{a}^{b} \alpha(s) d s=0, \sup |\alpha|>6 \pi(b-a)^{-1} \text { and sup }\left|\frac{d h}{d x}\right|=n
$$

where $R$ is a positive real number.

Let $u$ denote the vector field constructed in Example 3.3. We can define the following vector field on $\mathbb{R}^{2}: f(x, y)=\langle 1, \bar{u}(y) \cdot \alpha(x)\rangle$, where: 


$$
\bar{u}(y)=\left\{\begin{array}{cc}
n^{\prime}(y) u\left(n^{-1}(y)\right) & \text { for } y \in] c, d[. \\
u & \text { for } y \in \mathbb{P}-] c . \sigma[.
\end{array}\right.
$$

To assure that each ordit of any flow from $F I(f)$ crosses the set of orancheo points of $f$, we assume agditionally that there exist $a^{\prime} . b^{\prime}$ such that

$$
f_{a}^{b} \propto(s) d s>\sigma^{-1}(1)
$$

where $\sigma$ is defined in Example 3.3.

DEF INITION 4.5

We say that a vector field $v_{1}$ is a nod $f 2$ cat 2 on of $v_{0}$ and write $v_{1}=M O D(U . R) v_{0} 1 f$ there exist $\alpha, n, \lambda, \Omega$ as above such that $v_{1}=v_{0}$ outside $U$ and $v_{1}=f$ in $U$ in local coordinates given by $\lambda$.

To analyze properties of $v_{1}$ note that $f$ nas $F c$-oroperty: for anv $\phi \in F l(u)$, it generates the flow

$$
\varphi(t,(x, y))=\left(x+t, \phi\left(\int_{0}^{t} \alpha(x+s) d s, y\right)\right) .
$$

Vector fields $\langle 1,0\rangle$ and $f$ are different only inside the rectangle $\Omega$. It 15 easy to see that $R=] a, b[\times R(\bar{u})$ is the set of f-strong branched points. Condition (4.3) implies that the orbits of any flow generated by $f$, which pass across $\Omega$ have nonempty intersection with the set $R$. It is easy to see that flows generated by $f$ which are of the form (4.4) are conjugate with the unit flow $\mathbf{1}_{2}$ by the foll lowing homeomorphism $\Lambda$ :

$$
\Lambda(x, y)=\left(x, \phi\left(\int_{-\alpha}^{x} \alpha(x+s) d s, y\right)\right) \text {, for }(x, y) \in \mathbb{R}^{2} .
$$

\section{DEFINITION 4.6}

We say that a vector field $V$ has $\alpha$-property if for any point pex(v) there exists a connected neighborhood $U(p)$ and a local map $\lambda: U(p) \rightarrow \lambda(U(p)) \subseteq \mathbb{R}^{2}$ such that $V$ has the coordinates $\langle 1,0\rangle$ in the map $\lambda$.

Observe that if a vector field $V$ has the $\alpha$-property, $p$ and $U(p)$ are as in the above definition, then MOD(U(p), $\Omega) V$ has the $\alpha$-property. The operation $\operatorname{MOD}(U(p), R)$ depends on the choice of the local map $\lambda$. We can choose $\lambda$ in such a way that $p \in R(\operatorname{MOD}(U(p), R) \cup)$. In the following we shall always choose such a $\lambda$. STEP 2

We choose a countable dense set $P=\left\{p_{n}: n \in \mathbb{N}\right\}$ in $\mathbb{R}^{2}$ and start from the vector field $V_{1}$ with coordinates $\langle 1,0\rangle$ which obviously has a-property. Let $v_{n}$ denote the vector field obtained in $n$-th iteration. As the next 1 teration we take $v_{n+1}=\operatorname{MOD}\left(U\left(p_{n}\right), R_{n}\right) v_{n}$ if $p_{n} \in \overline{\mathcal{R}\left(V_{n}\right)}$ and $v_{n+1}=v_{n}$ otherwise.

LEMMA 4.7

Parameters $R_{n}$ of the MOD-operation can be chosen small enough, so that

a) the sequence $\left(V_{n}\right)$ converges uniformly on $\mathbb{R}^{2}$ (in the sense of the uniform convergence of coordinates in the canonical map in $\mathbb{R}^{2}$ ).

b) each of $v_{n}$ has the Fc-property. 
c) $R\left(V_{n+1}\right) \equiv R\left(V_{n}\right)$.

Proof. The distance $d_{n}$ between $v_{n}$ and $v_{n+1}$. defined as $\sup \left\{\left\|V_{n}(x)-V_{n+1}(x)\right\|: x \in\left[\mathbb{P}^{2}\right\}\right.$, is proportional to $n_{n}$ (with a constant depending on $n)$. so it is possible to make the series $\sum_{n=1} a_{n}$ convergent. D) and cl follow immediately from the construction. Q.E.D.

If $n$ is such that $v_{n+1} v_{n}$ then for any flow $\Phi_{n} \in I\left(V_{n}\right)$ we can construct, using (4.3), continuum of flows $\Phi_{n+1} \in F I\left(V_{n+1}\right)$ which are equal to $\Phi_{n}$ outside $U\left(p_{n}\right)$ ( In the sense of definition 4.4). The distance between $\Phi_{n}$ and $\Phi_{n+1}$ defined as $\sup \left\{\left\|\Phi_{n}(t, p)-\Phi_{n+1}(t, p)\right\|: t \in \mathbb{R}, p \in \mathbb{R}^{2}\right\}$ can be bounded by constant (depending on $n$ ) proportional to $\Omega_{n}$.

LEMMA 4.8

Farameters $R_{n}$ of the MOD-operation can de chosen small enough, so that any sequence $\left(\Phi_{n}\right.$ ) chosen as above converges uniformly to a flow on $\mathbb{R}^{2}$.

The proof follows from the above remarks.

PROFOSITION 4.9

If $n_{n}$ are chosen according to Lemmas 4.7 and 4.8 then the vector field $v=11 \mathrm{~m} v_{n}$ nas a dense set of strong branched points. The limit of any sequence ( $\Phi_{n}$ ' as in Lemma 4.8 is a flow generated by $v$.

Proof. Lemma 4.8 guarantees that $11 \mathrm{~m} \Phi_{n}$ is a flow on $\mathbb{R}^{2}$. From standard theorems about differential equations (see [5] Theorem 4.4) follows that $1 \mathrm{~m} \Phi_{n}$ is generated by $V$. It is easy to see from the construction, that using different flows from $F I(\bar{u})$ in any $\operatorname{MOD}\left(U\left(p_{n}\right), n_{n}\right)$-operation we obtain different $11 \mathrm{mits} 11 \mathrm{~m} \Phi_{n}$. It implies that $V$ has FC-property and any point of $P$ is a $V$-strong branched point. Q.E.D.

It can be proved that if the parameters $R_{n}$ are sufficiently small the set $R(V)$ is dense but different from $\mathbb{R}^{2}$. This completes Example 4.4.

REMARK 4.10

If the vector field $u$ used in MOD-operation, is replaced by the vector field constructed in Example 3.4 we can obtain a vector field $V^{*}$ such that $R\left(V^{*}\right)=R^{2}$, i.e. $\mathbb{R}^{2}$ is a strong branched set.

\section{GENERAL PROPERTIES DF VECTOR FIELDS AND FLOWS WITH FC-PROPERTY}

In this part we investigate sets of vector fields and flows having Fc-property. Those sets will be regarded as subsets of the spaces $F^{\circ}(M)$ and $F 1(M)$ respectively, which consist of all continuous vector fields and flows on a finite-dimensional manifold $M$. In order to avoid problems with non-integrable vector fields and with definition of the uniform convergence of vector fields we assume that all considered manifolds are compact.

PROPOSITION 5.1

If $M$ is an $n$-dimensional manifold and $n \geq 2$, then any continuous vector field on $M$ can be uniformly approximated by vector fields with Fc-property.

Proof: If the given vector field is smooth and does not vanish one can apply MOD-operation with a sufficiently small parameter $R$. If the field vanishes in an 
open set one can add to 1 t any field with Fi-property, multiplied by a sufficiently small constant. A continuous vector field can be uniformly approximated by smooth fields. B.E.D.

Let $k$ be any cardinal number; the set of continuous, bounded vector fields on $M$ with the $F k$-property will be denoted by G(M.k). The same way. If we consider the set $G_{1 n t}(M)$ of vector fields with nonempty, open sets of strong branched points (for instance $G_{1 n t}\left(\mathbb{R}^{2}, \infty\right.$ - see Remark 4.10), it can be similarly proved that $G_{1 n t}(M)$ is dense in $F^{\circ}(M)$. The sets $G(M, O)$ and $G(M, 1)$ are also dense in $F^{\circ}(M)$, it can be shown much more: $G(M, 1)$ is the set of second Baire category (see [3] pp. 119-121).

The space $F^{\circ}(M)$ may be in a natural way represented as a sum of disjoint sets $G(M, k)$, for $k \geq 0$. Unfortunately, for many dimensional (dim $M \geq 2$ ) manifolds authors do not know anything about the sets $G(M, k)$ for $k \geq 2$ and $k \leq N_{0}$; are these sets nonempty?

It was shown that on the $n$-dimensional manifolds, $n \geq 2$, there exist vector fields having open sets of strong branched points. For an arbitrary open set, approximating it by sums of rectangles, we get.

PROPOSITION 5.2

Every open subset $U d \mathbb{R}^{n}$, where $n>1$, is a branched set.

It is easy to see that the stronger result occurs: every open subset of the $n$-dimensional manifold, where $n>1$, is a branched set.

DEFINITION 5.3

For a compact manifold $M$ and given cardinal number $k$ we define the following sets:

$$
\begin{aligned}
& A_{q}(M, k)=U_{f \in G(M, k)}\{f\} \times F l_{q}(f) \\
& B_{q}(M)=U_{f \in F^{\circ}(M)}^{U}\{f\} \times F 1_{q}(f)
\end{aligned}
$$

Since $M$ is metrizable, then using the metric $p$ on $M$ we define a topology of above sets by the following metric:

$$
d\left(\left(f_{1}, \varphi_{1}\right),\left(f_{2}, \varphi_{2}\right)\right)=\left\|f_{1}-f_{2}\right\|_{0}+p_{0}\left(\varphi_{1}, \varphi_{2}\right)
$$

where $\|\cdot\|_{0}$ is the norm of the uniform convergence in $F^{\circ}(M)$, the set of all continuous vector fields on $M$, and $P_{0}$ denotes the norm:

$$
P_{0}\left(\varphi_{1}, \varphi_{2}\right)=\sup _{t \in \mathbb{R}} \sup _{x \in M} p\left(\varphi_{1}(t, x), \varphi_{2}(t, x)\right) .
$$

In what follows the topology defined by $d$ in those sets will be called the $c^{\circ}$-topology.

Before formulating results of this section, we give a short description of MOD1operation which which assigns to a quasi-flow $\psi$ a quasi-flow $\varphi$ having the Fc-property such that the $P_{0}(\varphi, \psi)$ can be arbitrarily small. Let $V$ be a continuous vector field on a manifold $M$ with the set $Z(V)$ of critical points diffe- 
rent from $M$. Suppose that $F l_{Q}(V)$ is not empty and let $\psi$ de a quasi-flow generated by $V$. It is oovious that for any $x e Z$ ( $V$ ) there exists a map (..U) of a nelghbourhood $U$ of $x$ such that $\psi$ is conjugate to the unit flow $1_{n}$ on $u^{\prime} 0_{\psi}(x)$ (1n the sense of definition 2.5). Without loss of generality we can assume that (U) contains the cube $[0.1]^{n}$. Let $\alpha$ be a nonnegative continuous function on $\mathbb{R}^{1}$ such that

(a) $x(\cdot)-1$ vanishes deyond a neighbornood of the Cantor set $\bar{c}$ on intervai $[0,1]$,

(b) $\int_{0}^{1}(\alpha(s)-1) d s<\delta$, where $\delta$ denotes a positive number.

(c) for any $x \in C, \quad(x)=1$.

Let $\psi$ ' be anv flow which in the map $(\lambda, U)$ has the following coordinates:

$$
\psi(t, x)=\left(p r_{1}(x)+\int_{a_{1}}^{D_{1}} \alpha(s) d s, p r_{2}(x), \ldots, p r_{n}(x)\right),
$$

where $x \in \mathbb{R}^{n}$. Let $\varphi$ denote the quasi-flow which is equal $\psi$ on $M-1 r O_{\psi}(x)$, and $\psi$ ' on $\operatorname{LrO}_{\psi}(x)$.

DEFINITION 5.4

We say that a map $\varphi_{1}: \mathbb{R} \times M \rightarrow M$ is a modification of quasi-flow $\psi$ and write $\varphi_{1}=\operatorname{MOD}(U, \delta) \psi$ if there exist $\alpha, \lambda, \varepsilon, U, \psi, \psi$ ' as above such that $\varphi_{1}=\psi$ outside $\operatorname{LrO}_{\psi}(x)$ and $\varphi_{1}=\psi^{\prime}$ in $\operatorname{Lro}_{\psi}(x)$.

It is easy to see that the map: $\varphi$ is a quasi-flow with a Fc-property, sufficlently close to $\psi: \quad 0(\varphi, \psi) \leq \varepsilon$, where $\varepsilon=S \delta$ and $S$ denotes a constant depending on $\lambda, \alpha$ and $U$.

THEOREM 5.5

Let $M$ be a compact smooth manifol a with Euler characteristic $\chi(M)$ different from zero. Suppose that $V$ is continuous vector field on $M$ and $\varphi_{n} F l_{q}(V)$. Then there exists a sequence of vector fields $V_{n}$ having the Fc-property and quasi-flows $\varphi_{n} \in l_{q}\left(V_{n}\right)$ which uniformly converge to $V$ and $\varphi$ respectively; $1 . e$. the set $A_{q}(M, C)$ is dense in $B_{q}(M)$ in $C^{\circ}$-topology.

Proof. Let $V$ be a continuous vector field on $M$ with nonempty set $F l_{q}(V)$. If $V$ vanishes in an open subset of $M$ theorem is obvious. In the other case let us consider an integer number $n$ and any quasi-flow $p \in F l_{q}(V)$. Since $x(M) \neq 0$, it follows from Poincare-Hopf theoren (see [B] 0.69 ), that $V$ has nonempty set $Z(V)$ of critical points. Let us choose a point $x \in Z(V)$ and open set $U$ such that - $(x) r y$ is disjoint from $Z(V)$.

Performing the MOD1-operation we obtain quasi-flow $\psi=$ MOD $1(U, 1 / n) \varphi$ which satisfies the condition

$$
\left\|V^{\prime}-V\right\|_{0}+P_{0}(\Psi, \varphi) \leq n^{-1}
$$

where $V$ 'denotes the vector field which generates the quasi-flow $\psi$. Since $n$ can be chosen arbitrarily large, the proof is complete. Q.E.D.

Since Euler characteristic $x\left(S^{k}\right)$ of $k$-dimensional sphere is equal $1+(-1)^{k}$, it follows immediately from theorem 5.5 that the set $A_{q}\left(S^{2 n}, c\right)$ is dense in $B_{q}\left(S^{2 n}\right)$ 
in $0^{0}-$ topologi.

Theorem 5.5 does not, in general, nold for manifolds with tuler characteristic zero. For example, since any vecto- field on $5^{1}$ with Fc-property nas uncruntade many of critical points isee section 3): this fact imolies that $A\left(S^{1} . C\right)$ is not dense in $B_{a}\left(S^{1}\right)$. There exist also nigher aimensional manitolds forr which ineorem 5.5 does not nold. Let us consider $\left.T^{2}=S^{1} \times 5^{1}=\exp (2 \pi 1 \alpha): x \in R\right) \times\{\exp (Z \pi 1 \beta): \beta \in R\}$. and the flow $\Phi$ naving the form $\Phi(t,(\alpha, \beta))=(\alpha+\Delta t+a t \cos \beta, \beta)$, where a and $\Delta$ are positive numbers. Let $v_{\Phi}$ denote the vector field which generates $\Phi$. It can be checked that the pair $\left(V_{\Phi}, \Phi\right)$ can not be uniformly approximated bv the vector fields and flows from $A_{q}(M, C)$. It appears, nowever, that $v_{\Phi}$ and $\Phi$ separately can be uniformly approximated by vector fields and flows with Fc-property. Note that this fact is more general:

PROFUSITION 5.6

If $M$ is a compact smooth manifold. then the set $\underset{V \in G(M, C)}{U} F q^{(V)}$ is a dense subset of $U F_{q}(V)$ in the $C^{\circ}$ topology, 1.e. any quasi-flow on $M$ can de uniformly $V \in F^{\circ}(M)$

approximated by quasi-flows with Fc-property.

The proof is analogous to the proof of Theorem 5.5 and will be omitted.

We can easily construct an analogous example of a vector field and a flow on Klein bottle $K^{2}$. Since for any two-dimensional compact manifold apart from $\$^{2}$ and $\mathrm{KK}^{2}$ Euler characteristic is negative, we can formulate:

COROLLARY 5.7

Suppose that $M$ is a two-dimensional compact manifold. Then the following conditions are equivalent:

1. Euler characteristic $\chi(M)$ is different from zero,

2. The set $A_{q}(M, C)$ is dense in $B_{q}(M)$ in $C^{\circ}$ topology,

3. $M=S^{2}$ and $M=K^{2}$.

\section{REFERENCES}

1. BATTY, C.J.K. Delays to flows on the real line. Preprint, 1979, unpub

2. BECK, A. Continuous Flows in the Plane, Springer-Verlag, Beriin, Hezdelberg, New York, 1974.

3. CHOQUET, G. Lectures on Analysis, W. A. Benjamin, Mathematical Lecture Series, 24 .

4. ENGELKING, R., SIEKLLCKI,K. Geometry and topology, PWN, Warsaw, 1981

5. HARTMAN, P. Ordinary Differential Equations, John Wiley and Sons, N. Y. , London, Sydney, 1964.

6. HARTMAN, P. A differential equation with nonunzque solutions. Amer. Ma Monthly, 70 (1963), PP. 255-259.

7. LAVRENTIEV, M. Sur une equation differentielle du premier ordre, Mat Zeit. 23 (1925), pp. 197-209.

8. SZlENK, W. Smooth dynamical systems, PWN Warsaw, 1982. 


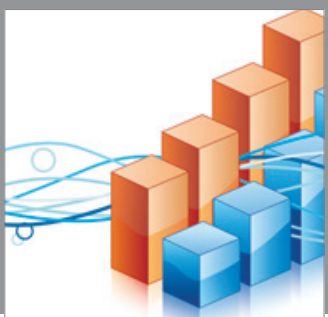

Advances in

Operations Research

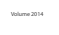

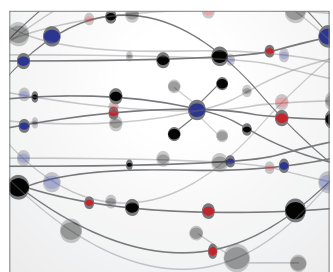

\section{The Scientific} World Journal
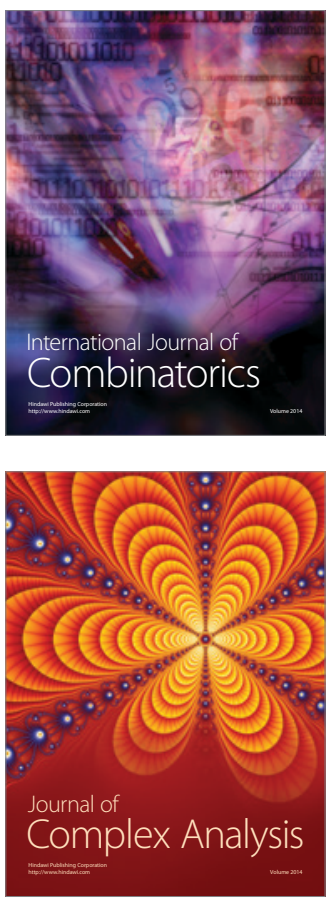

International Journal of

Mathematics and

Mathematical

Sciences
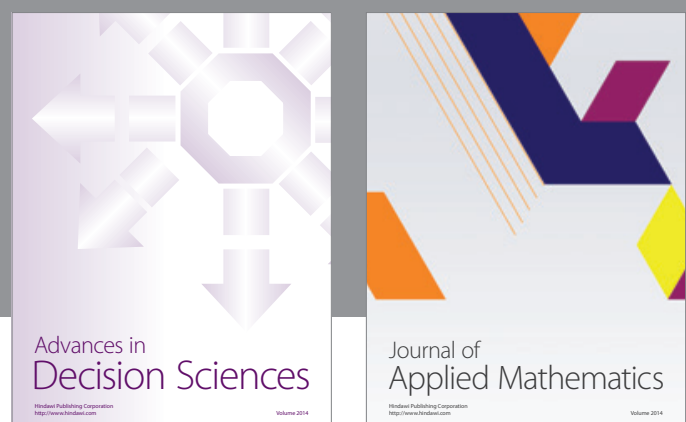

Journal of

Applied Mathematics
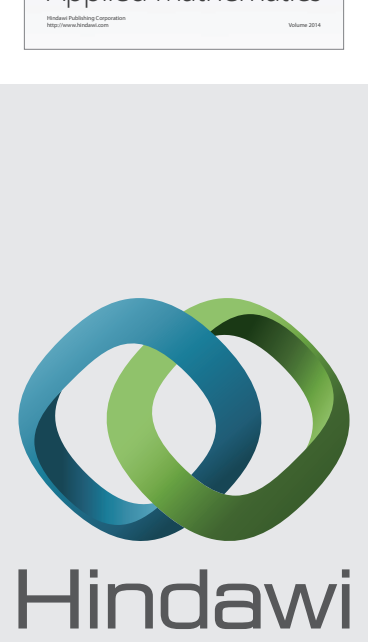

Submit your manuscripts at http://www.hindawi.com
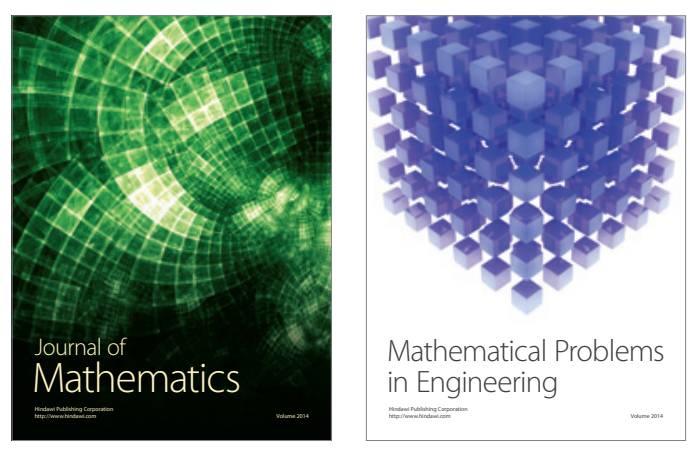

Mathematical Problems in Engineering
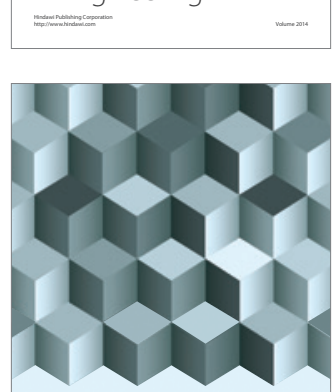

Journal of

Function Spaces
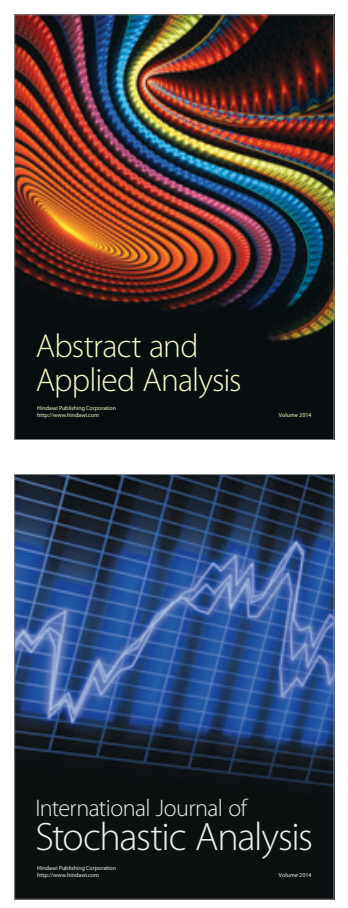

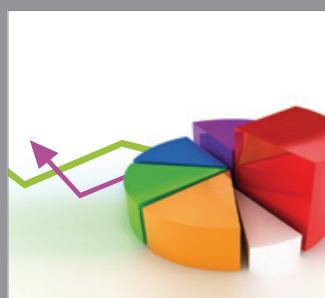

ournal of

Probability and Statistics

Promensencen
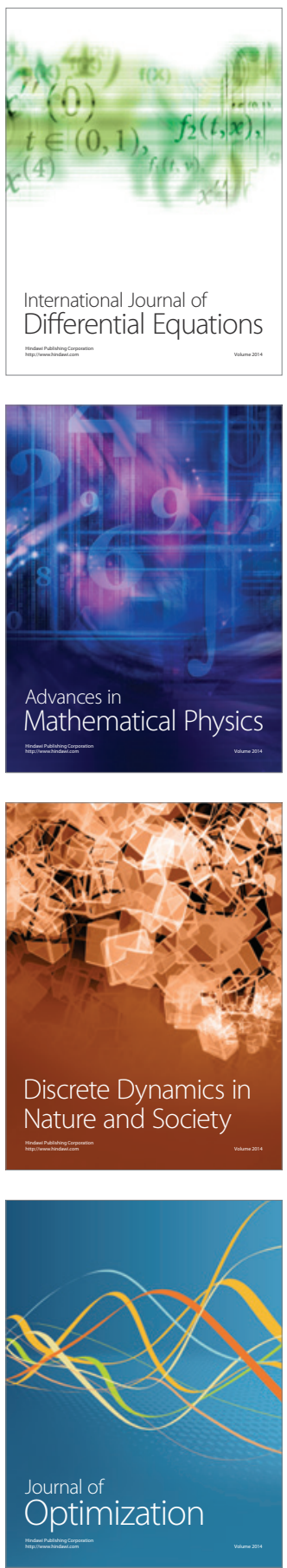